\title{
MFU-4 - A Metal-Organic Framework for Highly Effective $\mathrm{H}_{2} / \mathrm{D}_{2}$ Separation
}

\author{
Julia Teufel, Hyunchul Oh, Michael Hirscher,* Mohammad Wahiduzzaman, \\ Lyuben Zhechkov, Agnieszka Kuc, Thomas Heine, Dmytro Denysenko, and Dirk Volkmer
}

Deuterium is irreplaceable for many applications, like fusion reactions, nuclear-power production, neutron-scattering techniques, and isotope tracing. It is challenging to satisfy the world demand, as deuterium makes up only $0.0156 \%$ of the naturally occurring hydrogen isotopes..$^{[1]}$ Moreover, its extraction from isotope mixtures is difficult as the isotopes possess the same size and chemical properties. The conventional techniques for hydrogen/deuterium separation, like cryogenic distillation, the Girdler Sulfide process, thermal diffusion, and centrifugation are highly energy consuming, and therefore expensive. Furthermore, the selectivity of these techniques is less than $2.5 .^{\left[{ }^{[2]}\right.}$ At the end of the $20^{\text {th }}$ century, a new method of gaseous isotope separation by cryogenic gas adsorption in nanoporous structures, quantum sieving, was proposed. ${ }^{[3]}$ Adsorption isotherms for pure isotope gases have only been measured for a small number of porous materials, like activated carbons, zeolites, and metal-organic frameworks (MOFs), wherein the calculated $\mathrm{D}_{2} / \mathrm{H}_{2}$ uptake ratio at $77 \mathrm{~K}$ is used as a measure for the separation capability. However, the $\mathrm{D}_{2} / \mathrm{H}_{2}$ uptake ratios for all of these investigated structures are much less than 2 , and, therefore, they are no alternative to conventional separation methods. ${ }^{[4]}$ Here, we show that the separation performance can be greatly enhanced in an MOF with interconnected small and large cavities that are connected by a small aperture, whose size is close to the kinetic diameter of hydrogen. For this new material, the selectivity is investigated by way of pure $\mathrm{H}_{2}$ and $\mathrm{D}_{2}$ lowtemperature adsorption-isotherm measurements down to $50 \mathrm{~K}$ and, additionally, by a new method enabling the determination of the $\mathrm{D}_{2} / \mathrm{H}_{2}$ adsorption ratio in the presence of an isotope mixture. Both methods yield $\mathrm{D}_{2} / \mathrm{H}_{2}$ ratios much larger than those observed for other porous materials published up to now, and an even higher separation performance has been measured if

\author{
J. Teufel, H. Oh, Dr. M. Hirscher \\ Max Planck Institute for Intelligent Systems \\ Heisenbergstr. 3, 70569 Stuttgart, Germany \\ E-mail: hirscher@is.mpg.de \\ M. Wahiduzzaman, Dr. L. Zhechkov, Dr. A. Kuc \\ Prof. T. Heine \\ Jacobs University \\ School of Engineering and Science \\ Campus Ring 1, 28759 Bremen, Germany \\ D. Denysenko, Prof. D. Volkmer \\ Augsburg University \\ Institute of Physics \\ Universitätsstr. 1, 86159 Augsburg, Germany
}

kinetic factors are exploited. Our results clearly reveal the potential of highly advanced metal-organic frameworks for industrial isotope-separation processes and introduce a highly sensitive technique to determine the practical selectivity.

Metal-organic frameworks (MOFs) are crystalline hybrid solids consisting of inorganic connectors and organic linkers. In contrast to other porous materials, the 3D framework structure of an MOF is precisely tunable by choosing adequate precursors. ${ }^{[5]}$ Owing to their realizable ultrahigh porosity, MOFs offer an enormous specific surface area, which makes them very attractive for gas storage by physisorption on their inner surface. ${ }^{[6]}$ Under cryogenic conditions, isotopes exhibit differences in adsorption behavior caused by their different values of zero-point energy. If isotopes are adsorbed at low temperature in a framework, where the difference between the molecular size and the pore size becomes comparable to the de Broglie wavelength, the heavier isotope is more favorably adsorbed and can diffuse faster with respect to the lighter one. This phenomenon is referred to as quantum sieving ${ }^{[3]}$ and may be exploited for the separation of gaseous isotope mixtures like $\mathrm{H}_{2} / \mathrm{D}_{2}$. Only a few porous materials have been investigated with respect to their isotope adsorption. Although quantum-effect-induced differences in the adsorbed amount of hydrogen and deuterium could be observed, the obtained $\mathrm{D}_{2} / \mathrm{H}_{2}$ uptake ratio was just slightly larger than one, implying no relevance for technical use; in one exception, extraordinary large selectivity values of up to 5.8 for an MOF have been reported. ${ }^{[7]}$ The selectivity corresponds to the $\mathrm{D}_{2} / \mathrm{H}_{2}$ adsorption ratio in the presence of a 1:1 gas mixture. However, these high selectivity values of the Cu-MOF are only calculated by applying ideal adsorption solution theory to pure gas-adsorption isotherms measured at $40 \mathrm{~K} .{ }^{[7]}$ The experimental $\mathrm{D}_{2} / \mathrm{H}_{2}$ ratio for the pure gases is only around 2 at $40 \mathrm{~K}$ for this $\mathrm{Cu}-\mathrm{MOF}$. We propose MFU-4 ${ }^{[8]}$ as a Zn-based MOF featuring exceptionally high thermal and hydrolytic stability due to Kuratowski type secondary building units. The MFU-4 structure possesses a pore system of alternating small and large cavities connected by a small square-shaped aperture formed by four chlorine atoms (Figure 1a). Any molecule becoming adsorbed inside the MFU-4 framework has to pass the small cavity and the aperture in order to reach the large cavity, which leads to an experimentally observed gating effect. ${ }^{[9]}$ As the small cavity is only $3.88 \AA$ in diameter and the aperture is as narrow as $2.52 \AA$ (estimated using the van der Waals radii of the $\mathrm{Cl}$ atoms), larger molecules, such as nitrogen, are completely excluded from the framework at low temperatures, whereas smaller molecules like hydrogen and water can still enter. ${ }^{\left[{ }^{9]}\right.}$ For the quantum-sieving experiments in MFU-4, the small cavity and the aperture act as separation 


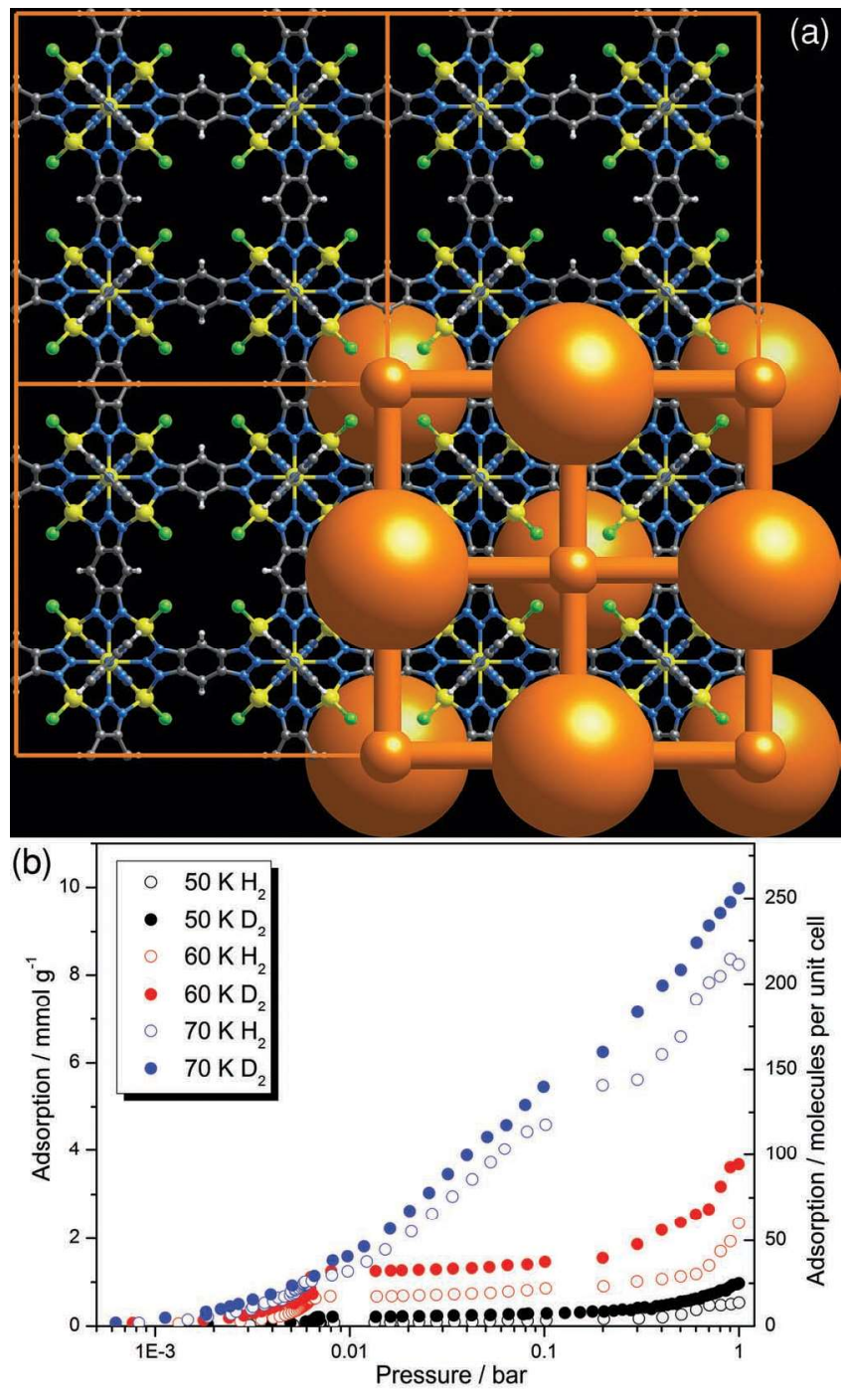

Figure 1. a) Crystal-structure packing model of the MFU-4 framework ${ }^{[9]}$ showing the arrangement of interconnected large and small cavities (inner diameters of $11.94 \AA$ and $3.88 \AA$ ) separated by narrow apertures of $2.52 \AA$. b) Low-temperature adsorption isotherms for pure hydrogen and pure deuterium measured up to 1 bar at $50 \mathrm{~K}$ (black), $60 \mathrm{~K}$ (red), and $70 \mathrm{~K}$ (blue). The open symbols refer to hydrogen and the closed symbols to deuterium.

gates, whereas the large cavity of $11.94 \AA$ (diameter) offers the surface area for storing large amounts of deuterium.

The narrow apertures between the small and the large cavities form a barrier, estimated to be $\approx 100 \mathrm{meV}$ on the BornOppenheimer surface for a perfect and stiff network (see Supporting Information). Interestingly, the lowest energy profile for hydrogen to pass from one cavity to the other will be obtained if $\mathrm{H}_{2}$ is perpendicular to the cavity, that is, that hydrogen is in plane with the four $\mathrm{Cl}$ atoms defining the MFU-4 aperture in the transition state. The barrier at the gate is based on the Pauli repulsion between the $\mathrm{Cl}$ atoms and $\mathrm{H}_{2}$, and it is pronounced, making classical diffusion impossible, and not even tunneling would allow a noticeable gas penetration into a perfect and stiff network. However, as shown by $\mathrm{H}_{2}$ and $\mathrm{D}_{2}$ adsorption isotherms, measured at temperatures of $50 \mathrm{~K}$ and above, $\mathrm{H}_{2}$ and $\mathrm{D}_{2}$ penetrate the MFU-4 framework.

As the interaction of $\mathrm{H}_{2}$ with the gate is governed by Pauli repulsion, which depends exponentially on the distance between the interacting entities, small structural changes cause large variations in the barrier. Those structural changes are possible by lattice vibrations, and indeed we compute that the lowest-energy optical phonons occur in MFU-4 at energies corresponding to $45 \mathrm{~K}$ to $85 \mathrm{~K}$ (see supporting information). Those phonons relate to vibrational modes involving the four $\mathrm{Cl}$ atoms that define the aperture size between the small and large cavities, and hence control the only channel for hydrogen to diffuse through the framework. Therefore, we suggest that the gating behavior is controlled by the temperature through lattice vibrations.

These theoretically derived conclusions are supported by the low-temperature pure-gas-adsorption isotherms measured at $50 \mathrm{~K}, 60 \mathrm{~K}$, and $70 \mathrm{~K}$ up to 1 bar (Figure $1 \mathrm{~b}$ ). The uptake values are given per unit cell containing four small and four large pores. The uptake for hydrogen and deuterium grows with increasing adsorption temperature from $13.6 \mathrm{H}_{2}$ and $24.9 \mathrm{D}_{2}$ per unit cell at $50 \mathrm{~K}$ to $209.8 \mathrm{H}_{2}$ to $254.1 \mathrm{D}_{2}$ per unit cell at $70 \mathrm{~K}$ (see Supporting Information, Table S2). Usually, the adsorption isotherms indicate decreasing gas uptake with rising adsorption temperature. However, the large diffusion barrier, which is a prerequisite for quantum sieving, does not allow the measurement of equilibrium isotherms at low temperatures in a reasonable time.

Thus, the increasing uptake with rising adsorption temperature can be attributed to the temperature triggered opening of the aperture that reduces the kinetic barrier and therefore facilitates the gas uptake. If adsorption isotherms are measured for the standard temperature of $77 \mathrm{~K}$, and for higher loading temperatures where the MFU-4 structure is expected to be open, MFU-4 also shows the trend of declining uptake with rising adsorption temperature. ${ }^{[9]}$ In the work by Denysenko et al., ${ }^{[9]}$ the heat of adsorption is denoted as $7 \mathrm{kJmol}^{-1}$ over a wide range of surface coverage which is in agreement with other porous materials that offer pores below the kinetic diameter of nitrogen. ${ }^{[10]}$ The low-temperature adsorption isotherms measured at $50 \mathrm{~K}$ yield a maximum $\mathrm{D}_{2} / \mathrm{H}_{2}$ uptake ratio of 4.1 at 7 mbar, whereas at $70 \mathrm{~K}$, the maximum $\mathrm{D}_{2} / \mathrm{H}_{2}$ ratio of 1.7 occurs at 1.8 mbar. Hence, the maximum $\mathrm{D}_{2} / \mathrm{H}_{2}$ uptake ratio decreases with increasing adsorption temperature and shifts to lower pressures. To the best of our knowledge, the $D_{2} /$ $\mathrm{H}_{2}$ uptake ratios of MFU-4 are the largest values ever measured by pure-gas-adsorption isotherms.

After these encouraging results, we wanted to determine the selectivity (i.e., access directly the separation performance by applying an isotope mixture). The selectivity is assumed to be larger than the $\mathrm{D}_{2} / \mathrm{H}_{2}$ uptake ratios in the equilibrium adsorption experiments for two reasons. Firstly, the isotopes can compete directly for the adsorption sites that favor deuterium. Secondly, the kinetic nature of the quantum-sieving phenomenon (i.e., the higher diffusivity of the heavier isotope) can be exploited and the corresponding non-equilibrium selectivity values after short gas-exposure times will add to the deuterium enrichment in the adsorbed state. Thus, we present the first 

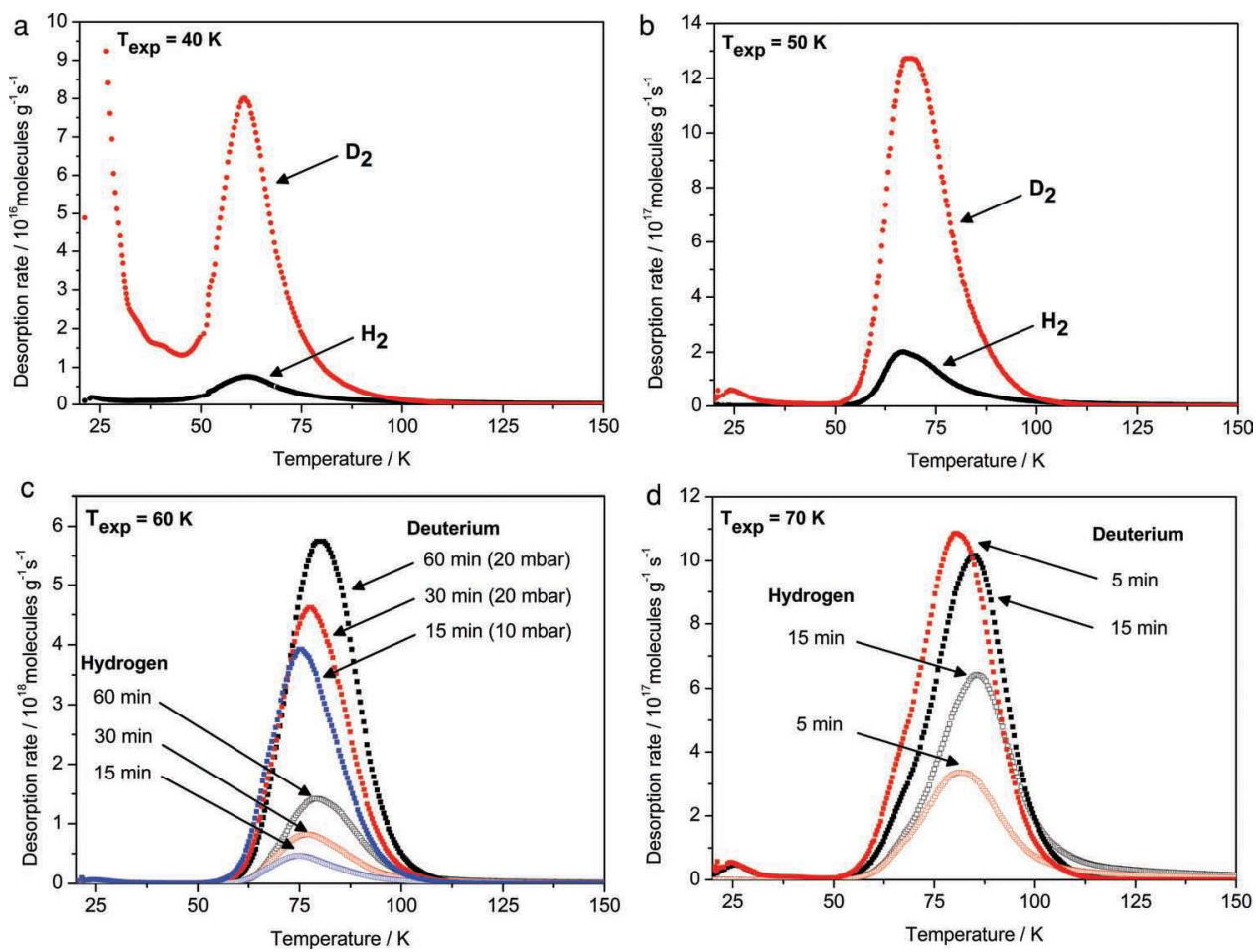

Figure 2. Hydrogen and deuterium thermal-desorption spectra obtained after exposure to a 1:1 mixture at: a) $40 \mathrm{~K}$, b) $50 \mathrm{~K}$, c) $60 \mathrm{~K}$, and d) $70 \mathrm{~K}$. The desorption spectra after exposure at $40 \mathrm{~K}$ and $50 \mathrm{~K}$ were measured for a fixed exposure time of $1.5 \mathrm{~h}$. For gas exposure at $60 \mathrm{~K}$ and $70 \mathrm{~K}$, the TDS experiments were performed for different exposure times as indicated in graph c) and $\mathrm{d}$ ).

direct measurement of non-equilibrium selectivity $S_{\text {neq }}(P, T, t)$, a function of exposure time $t$, by applying $\mathrm{H}_{2} / \mathrm{D}_{2}$ isotope mixtures in a home-made and calibrated thermal-desorption spectroscopy (TDS) apparatus. In TDS, the sample is exposed to a defined gas atmosphere in an ultrahigh vacuum (UHV) chamber for a chosen exposure time. Afterwards, the sample is rapidly cooled to the boiling temperature of the adsorbed gas and the remaining gas molecules are pumped off until a high vacuum is reached again. Finally, a linear heating ramp is applied in order to thermally activate desorption. The desorbing gas is continuously detected using a mass spectrometer, recognizing a pressure increase in the sample chamber when gas desorbs. The area under the desorption peak is proportional to the desorbing amount of gas, which can be quantified after careful calibration of the TDS apparatus. In this type of experiment, the selectivity is given as the ratio between the amounts of desorbed $\mathrm{D}_{2}$ to $\mathrm{H}_{2}$. Physically, when the sample is exposed to a hydrogen-isotope mixture at low temperature, within the scope of a TDS experiment, hydrogen and deuterium molecules penetrate the framework and adsorb inside. However, when quantum effects occur, deuterium diffuses faster than hydrogen and therefore the isotopes penetrate the framework at different rates. The penetration rate depends on the exposure temperature. The adsorbed amount of an isotope depends on the exposure temperature and the exposure time. Hence, the selectivity values obtained by a TDS experiment with isotope mixtures depend on the exposure temperature and time, and higher selectivity values can be achieved if one takes advantage of kinetic factors. MFU-4 was exposed to a 10 mbar $1: 1 \mathrm{H}_{2} / \mathrm{D}_{2}$ isotope mixture at $40 \mathrm{~K}$ and $50 \mathrm{~K}$ for $1.5 \mathrm{~h}$ (Figure 2a,b).

The thermal desorption spectra show a low-temperature signal centered at $25 \mathrm{~K}$, which is only due to liquefied deuterium and gas adsorption at the outer surface. Only the desorption signal starting from $50 \mathrm{~K}$ can be related to the molecular adsorption inside the MFU-4 framework. Generally, the number of desorption maxima is equal to the number of energetically different adsorption sites. However, in MFU-4, the gas molecules in the small and large cavities cannot desorb independently due to the alternating cavity structure. Therefore, only one desorption maximum is visible. As TDS is more sensitive than the volumetric adsorption measurement, a low gas uptake of less than one molecule per unit cell is already measurable after loading the MFU-4 sample at $40 \mathrm{~K}$. The selectivity measured by TDS also decreases with increasing temperature, from 6.9 to 5.8 at $40 \mathrm{~K}$ and $50 \mathrm{~K}$, respectively (see Supporting Information, Table S3). The gas uptake is drastically increased when the loading temperature rises from $40 \mathrm{~K}$ to $50 \mathrm{~K}$. The hydrogen uptake increases by a factor of nearly 20 and the deuterium uptake by more than 16 , which is related to the opening of the aperture by phonon vibrations above $45 \mathrm{~K}$. In order to reveal the dependence of the selectivity on the exposure time, TDS experiments with an exposure to a $1: 1 \mathrm{H}_{2} / \mathrm{D}_{2}$ isotope mixture at $60 \mathrm{~K}$ and $70 \mathrm{~K}$ were performed for different exposure times (Figure 2c,d). At $60 \mathrm{~K}$ the selectivity drops from 7.5 to 3.7 after $15 \mathrm{~min}$ and $1 \mathrm{~h}$ exposure, respectively. When the gas exposure is carried out at $70 \mathrm{~K}$, the selectivity drops even faster from 2.8 to 1.4 after $5 \mathrm{~min}$ 


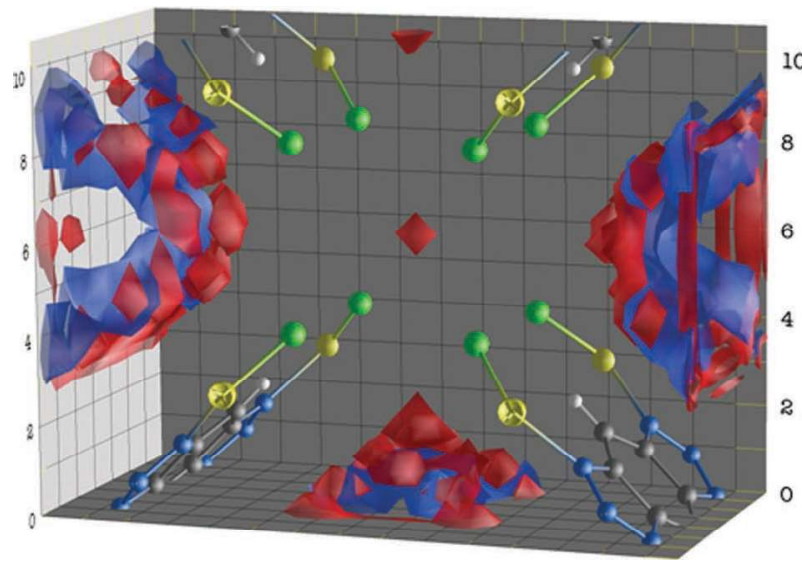

Figure 3. Local density differences $n\left(\mathrm{D}_{2}\right)-n\left(\mathrm{H}_{2}\right)$ in a box with the same number of $\mathrm{D}_{2}$ and $\mathrm{H}_{2}$ particles $n\left(T=77 \mathrm{~K}, n=8.512 \times 10^{-4} \mathrm{~m}^{3} \mathrm{~mol}^{-1}\right)$. The red areas indicate $a D_{2}$ excess close to the cavity surface and the aperture; the blue areas indicate a $\mathrm{H}_{2}$ excess inside the cavities.

and 15 min exposure, respectively. Hence, $D_{2}$ is preferred mainly in the first adsorption step, whereas for longer exposure times, equilibrium is approached, leading to a decrease in selectivity, which is even faster for higher exposure temperatures. The quantum-sieving effect in MFU-4 can be easily understood if the hydrogen and deuterium adsorption probability densities are compared with each other (Figure 3). For this purpose, we carried out quantum-mechanical adsorption calculations showing that $\mathrm{D}_{2}$ forms a quantum liquid at the cavity surface, while $\mathrm{H}_{2}$ shows a stronger probability to be adsorbed inside the cavities. These observations are in line with those of traditional quantum sieving and of recent observations in other metal-organic frameworks. ${ }^{[3,11]}$ As the $D_{2}$ density is high when close to the apertures, this gas will enter the framework in excess once penetration is possible due to the assistance of low-energy vibrational modes at $\approx 50 \mathrm{~K}$ and above. The preferred $\mathrm{D}_{2}$ adsorption of the framework lowers the $\mathrm{D}_{2}$ partial pressure in the exposed gas mixture, leading to a higher $\mathrm{H}_{2}$ concentration on the surface. Consequently, in time, larger amounts of $\mathrm{H}_{2}$ can enter the framework until a mixture equilibrium is reached inside the cavities. $\mathrm{As}_{2}$ and $\mathrm{D}_{2}$ occupy different positions in the cavities, an equilibrium of $\mathrm{H}_{2}$ and $\mathrm{D}_{2}$ is expected that will reduce the selectivity in disfavor of the anticipated sieving effect. These considerations must be taken into account for pursuing any practical applications, and a continuous gas flow is suggested to maintain a high selectivity.

In conclusion, this work emphasizes the great potential of advanced MOF materials for separating light gas isotope mixtures. MFU-4, an MOF with an alternating bimodal cavity structure, exhibits the highest $\mathrm{D}_{2} / \mathrm{H}_{2}$ uptake ratio of 4.1 determined experimentally, so far. The practical maximum selectivity of 7.5 for a $1: 1 \mathrm{H}_{2} / \mathrm{D}_{2}$ isotope mixture implies a possible alternative to the conventional isotope-separation techniques. Effective isotope separation in MOFs can be achieved in the presence of kinetic barriers that enable the separation by exploiting the kinetic nature of the quantum-sieving phenomenon. The additional presence of large cavities in the MFU-4 structure offers the required surface area for physisorption of deuterium molecules inside the framework.

\section{Experimental Section}

Sample Preparation: MFU-4 was synthesized according to Biswas et al. ${ }^{[8]}$ Dimethylformamide (DMF) solvent molecules were removed at $280^{\circ} \mathrm{C}$ in vacuum.

Isotherm Measurements: The hydrogen and deuterium adsorption isotherms of MFU-4 from $70 \mathrm{~K}$ down to $20 \mathrm{~K}$ were measured using laboratory-designed volumetric adsorption equipment with a temperature-controlled cryostat. Around $23 \mathrm{mg}$ of MFU-4 was activated, overnight, at $420 \mathrm{~K}$. The sample was evacuated under $1 \mathrm{mPa}$ at room temperature (RT) overnight prior to each measurement. For the laboratory-designed cryostat, the temperature control was calibrated by measuring the liquefaction pressure for hydrogen and nitrogen in the empty sample chamber at various temperatures.

TDS: The TDS experiments were performed in a home-made device with $6.92 \mathrm{mg}$ of MFU-4 powder. The sample chamber was a UHV chamber where the sample holder was screwed tightly to a Cu block, which could be cooled using an LHe cryostat and heated using a heating spiral. The MFU-4 powder was activated at $420 \mathrm{~K}$. For the TDS experiment, the sample was typically exposed to a $10 \mathrm{mbar} 1: 1 \mathrm{H}_{2} / \mathrm{D}_{2}$ mixture at the given exposure temperatures. Owing to the high deuterium uptake at $60 \mathrm{~K}$ and the long exposure times, 20 mbar was used to ensure a constant composition of the gas mixture. The exposure time at $40 \mathrm{~K}$ and $50 \mathrm{~K}$ was $1.5 \mathrm{~h}$. For loading at $60 \mathrm{~K}$ and $70 \mathrm{~K}$, the exposure times were varied and are given in the corresponding diagrams. After loading the sample at the exposure temperature, it was cooled down under a gas atmosphere to $20 \mathrm{~K}$. The gas molecules that had not been adsorbed were pumped off until HV was reached again. Then, a heating ramp of $0.1 \mathrm{~K} \mathrm{~s}^{-1}$ was started from $20 \mathrm{~K}$ to $300 \mathrm{~K}$. The gas coming out was detected continuously using a quadrupole mass spectrometer. In order to quantify the mass-spectrometer signal, the TDS device had been calibrated using a PdCe alloy ${ }^{[12]}$ (see Supporting Information).

Theory: The crystal structure of MFU-4 was fully optimized within density-functional theory (DFT), employing two different models, a periodic boundary condition (PBC) and a cluster $(C L)$ approach, thus having the possibility of comparing strongly different setups. PBC calculations were carried out using CRYSTAL09[13] with the explicit sampling of 29 distinct $k$ points in the irreducible Brillouin zone, a Perdew-Burke-Ernzerhof (PBE) exchange-correlation functional, ${ }^{[14]}$ and Gaussian-type basis functions (Cl_86-311G_apra_1993, Zn_86411d31G_jaffe_1993, H_5-11G*_dovesi_1984, N_6-21G*_dovesi_1990, C_6-21G*_catti_1993, and O_8-411_towler_1994). The CL calculations were carried out using Amsterdam density functional (ADF), ${ }^{[15]}$ employing Slater type all-electron basis functions (double zeta with polarization function basis set (DZP) for $\mathrm{C}, \mathrm{H}, \mathrm{O}$, and $\mathrm{N}$ and triple ZP (TZP) for Zn). We modeled the fully saturated cavity of MFU-4, leading to very similar structural properties as the $\mathrm{PBC}$ approach. As $\mathrm{H}_{2}$ adsorption is dominantly a London dispersion interaction, we used the B88LYP-D exchange-correlation functional. ${ }^{[16]}$

Quantum-liquid density functional theory (QLDFT ${ }^{[17]}$ allowed the study of $\mathrm{H}_{2}$ adsorption incorporating quantum effects and treated the hydrogen molecules as shapeless spherical particles interacting with the host material. We used the LIE-1 functional and the same details as in ref. ${ }^{[17]}$ and, for the hydrogen-framework interaction, the forcefield of Han et al., ${ }^{[18]}$ parameterized on extensive MP2 data and adopted to the QLDFT approach, was used. We used a cubic simulation box with $a=21.697 \AA$, $T=77 \mathrm{~K}$ and an average molar gas density of $8.512 \times 10^{-4} \mathrm{~m}^{3} \mathrm{~mol}^{-1}$. 


\section{Acknowledgements}

The authors are thankful for financial support from DFG SPP 1362; part of this research was supported by the European Commission through an ERC Starting Grant (GA 256962). We thank Dr. Andreas Mavrantonakis and Prof. Freek Kapteijn for helpful discussions.

[1] N. N. Greenwood, A. Earnshaw, Chemistry of the Elements, Elsevier Butterworth-Heinemann, Amsterdam/Heidelberg 2005.

[2] H. K. Rae, Separation of Hydrogen Isotopes, Vol. 68, American Chemical Society, Washington D.C., 1978.

[3] J. J. M. Beenakker, V. D. Borman, S. Y. Krylov, Chem. Phys. Lett. 1995, 232, 379-382.

[4] a) B. Chen, X. Zhao, A. Putkham, K. Hong, E. B. Lobkovsky, E. J. Hurtado, A. J. Fletcher, K. M. Thomas, J. Am. Chem. Soc. 2008 130, 6411-6423; b) X. Z. Chu, Y. P. Zhou, Y. Z. Zhang, W. Su, Y. Sun, L. Zhou, J. Phys. Chem. B 2006, 110, 22596-22600; c) T. X. Nguyen, H. Jobic, S. K. Bhatia, Phys. Rev. Lett. 2010, 105; d) X. B. Zhao, S. Villar-Rodil, A. J. Fletcher, K. M. Thomas, J. Phys. Chem. B 2006 110, 9947-9955.

[5] M. Eddaoudi, J. Kim, N. Rosi, D. Vodak, J. Wachter, M. O'Keeffe, O. M. Yaghi, Science 2002, 295, 469-472.

[6] a) S. Cavenati, C. A. Grande, A. E. Rodrigues, Ind. Eng. Chem Res. 2008, 47, 6333-6335; b) M. Hirscher, B. Panella, B. Schmitz, Microporous Mesoporous Mater. 2010, 129, 335-339; c) Y. Li,
R. T. Yang, Langmuir 2007, 23, 12937-12944; d) M. J. Manos, M. S. Markoulides, C. D. Malliakas, G. S. Papaefstathiou, N. Chronakis, M. G. Kanatzidis, P. N. Trikalitis, A. J. Tasiopoulos, Inorg. Chem. 2011, 50, 11297-11299.

[7] D. Noguchi, H. Tanaka, A. Kondo, H. Kajiro, H. Noguchi, T. Ohba, H. Kanoh, K. Kaneko, J. Am. Chem. Soc. 2008, 130, 6367-6372.

[8] S. Biswas, M. Grzywa, H. P. Nayek, S. Dehnen, I. Senkovska, S. Kaskel, D. Volkmer, Dalton Trans. 2009, 6487-6495.

[9] D. Denysenko, M. Grzywa, M. Tonigold, B. Streppel, I. Krkljus, M. Hirscher, E. Mugnaioli, U. Kolb, J. Hanss, D. Volkmer, Chem. Eur. J. 2011, 17, 1837-1848.

[10] B. Schmitz, I. Krkljus, E. Leung, H. W. Hoffken, U. Muller M. Hirscher, ChemSusChem 2010, 3, 758-761.

[11] a) P. Kowalczyk, P. A. Gauden, A. P. Terzyk, J. Phys. Chem. B 2008 112, 8275-8284; b) K. Sillar, A. Hofmann, J. Sauer, J. Am. Chem. Soc. 2009, 131, 4143-4150.

[12] M. Ege, H. Kronmuller, J. Alloys Compd. 1997, 261, 231-241.

[13] R. Dovesi, V. R. Saunders, C. Roetti, R. Orlando, C. M. Zicovich-Wilson, F. Pascale, B. Civalleri, K. Doll, N. M. Harrison, I. J. Bush, P. D'Arco, M. Llunell, CRYSTAL09 User's Manual, University of Torino, Torino 2009.

[14] J. P. Perdew, K. Burke, M. Ernzerhof, Phys. Rev. Lett. 1996, 77 3865-3868.

[15] Scientific Computing \& Modelling Amsterdam, www.scm.com (accessed October 2012).

[16] S. Grimme, J. Antony, S. Ehrlich, H. Krieg, J. Chem. Phys. 2010, 132.

[17] S. Patchkovskii, T. Heine, Phys. Rev. E 2009, 80.

[18] S. S. Han, S. H. Choi, W. A. Goddard, J. Phys. Chem. C 2010, 114, 12039-12047. 